\title{
Economic evaluation of vaccination against influenza in the elderly: an experience from a population-based influenza vaccination program in Taiwan
}

\author{
Sen-Te Wang ${ }^{\mathrm{a}, \mathrm{b}}$, Long-Teng Lee ${ }^{\mathrm{b}, \mathrm{c}}$, Li-Sheng Chen ${ }^{\mathrm{a}}$, Tony Hsiu-Hsi Chen ${ }^{\mathrm{a}, *}$ \\ ${ }^{a}$ Institute of Preventive Medicine, College of Public Health, National Taiwan University, Room 207, No. 19, Hsuchow Road, Taipei, Taiwan \\ ${ }^{\mathrm{b}}$ Department of Family Medicine, Taipei Medical University Hospital, Taipei, Taiwan \\ ${ }^{\mathrm{c}}$ Department of Family Medicine, National Taiwan University, Hospital, Taipei, Taiwan
}

Received 17 April 2004; accepted 11 October 2004

Available online 5 November 2004

\begin{abstract}
Due to viral strains, influenza season, and consultations and admission rates varying from country to country, the continued economic evaluation of influenza vaccination for the elderly people aged 65 years and above is paramount, particularly in areas with dense population. Efficacy and cost-effective analysis of influenza vaccination in reducing all-cause mortality and hospitalization was therefore elucidated based on a prospective and population-based study targeted to 226,997 elderly people aged 65 years and above residing in Taipei county, Taiwan between 1 October 2000 and 31 March 2001. Vaccination against influenza for the elderly persons can lead to a 29\% (95\% CI: 23-35\%) significant reduction of all-cause deaths. Approximately, 20\% (95\% CI: 9-30\%) significant reduction in hospitalization was observed for average-risk group but 4\% (95\% CI: -4-11\%) non-significant reduction for high-risk group. Community-based influenza vaccination program for elderly people aged 65 years and above was demonstrated to be effective in reducing mortality in all elderly people but not significantly in reducing hospitalization. Universal vaccination program for the elderly people seems cost-effective in averting death or gaining life years. (C) 2004 Elsevier Ltd. All rights reserved.
\end{abstract}

Keywords: Influenza vaccination; Efficacy; Cost-effectiveness

\section{Introduction}

The effectiveness of influenza vaccine in reducing deaths and hospitalization in the elderly has been corroborated by several cohort, case-control studies [1-4], and a randomized controlled trial [5]. Despites these evidence-based findings, the opinion regarding the immunization policy for influenza vaccine is still diversified and may vary from country to country. For example, even a body of evidence on effectiveness of immunization against influenza there are important differences of opinion in Europe and North America. Selection of specific immunization policy in each country may largely depend on the target population of inter-

\footnotetext{
* Corresponding author. Tel.: +886 2 23587620; fax: +886 223587707.

E-mail address: stony@episerv.cph.ntu.edu.tw (T.H.-H. Chen).
}

est, ethnic groups, geographic areas, and epidemic strain of virus. In addition, when healthcare providers are faced with increasingly aging population, costly new technologies, and greater awareness and expectation of population given limited sources, economic appraisal is desperately required to offer an opportunity to compare the new immunization initiative with existing health programs. This is particularly important when the immunization policy is targeted to both high-risk and non-high risk elderly people because the latter group may represent a large proportion of elderly people and need enormous costs even larger benefit is achieved. Furthermore, as suggested by Nicholson [6], economic appraisal may be needed country by country because consultation and admission rates for pneumonia and influenza and whether augmented costs (extra healthcare expenditure for the extra years of life produced by an inter- 
vention) should be considered vary from country to country.

As health policy-makers in Taiwan are now faced with increasingly aging population and the competition among costly emerging technologies or different population-based screening programs for cancer and chronic diseases whether large-scale influenza vaccination for the elderly people take first priority is largely dependent on evidence-based and economic evaluation. The previous study in southern Taiwan has demonstrated the effectiveness of influenza vaccination [7]. However, numbers of subjects recruited in this study were very small compared with other earlier studies in western countries. Furthermore, annual influenza activity, consultation rates, admission rates, diagnostic criteria also varies area to area in Taiwan. The evidence supporting universal immunization against influenza for approximately 1,600,000 elderly people aged 65 years or older is still tenuous in Taiwan. We therefore launched a large-scale vaccination program for approximately 230,000 elderly persons in the community in northern Taiwan during the epidemic period in 2000. Based on this population-based study, we aimed to assess the efficacy of vaccination program on the rate of all-cause mortality and hospitalization attributed to influenza and complications of influenza, including pneumonia, acute and chronic respiratory diseases, and congestive heart failure [8]. Costeffectiveness analysis was also performed to assess whether the expanded vaccination program for all elderly persons was cost-effective.

\section{Methods}

\subsection{Background information}

Since October 2000, Taipei county, the largest one in Taiwan, has initiated a large-scale influenza vaccination for the elderly persons aged 65 years or older. These include 226,997 subjects dwelling in this county obtained from population registry. This forms the target population in this study. The county consists of 29 townships. Each township has embarked on the vaccination at the same time. Most participants were vaccinated between 1 October and 31 December 2000. High-risk group was taken as the first priority and the average-risk group as the second priority.

\subsection{Study design and data linkage}

A prospective study design was devised to classify the target population into two groups, the vaccinated group and the unvaccinated group. Outcome measurements included all-cause mortality and hospitalization attributed to complications related to influenza virus infection. The major categories included pneumonia and influenza (codes 480-487 in the International Classification of Diseases, Ninth Revision, Clinical Modification [ICD-9-CM]), respiratory disease (ICD-9-CM codes 460, 462, 465, 466, 480-487, 490-496,
500-518), and congestive heart failure (CHF, ICD-9-CM codes 428). These diagnoses were chosen according to the previously identified categories of complications associated with influenza [8]. To ascertain death, the target population list was linked with Taiwan mortality registry that includes all deaths toward the end of 2001. Hospitalization data were also accrued by linking the targeted population with national health insurance claimed data covering residents aged over 65 years in Taipei county. Outcomes collected were limited to the influenza season.

\subsection{Vaccine strain}

The vaccine strain used was consistent with the possible epidemic strain predicted by World Health Organization, including A/Moscow/10/99 (H3N2)-like strain, A/New Caledonia/20/99(H1N1)-like strain, B/Beijing/184/93-like strain, all with $15 \mu \mathrm{g}$ of hemagglutinin. As we had a smallscale breakout of B type, expressed as Beijing/184/93-like strain proven by center for disease control [9], between late December 2000 and January 2001, this confirmed that the vaccine strain was similar to the epidemic strain.

\subsection{Influenza season}

In accordance with the criteria of CDC in the USA [10], the influenza seasons were defined on the basis of active influenza-surveillance data from CDC in Taiwan [11]. The morbidity of influenza started to rise from the beginning of December, reached the peak in January and showed a plateau by the end of March. The influenza season in this study was therefore defined between 1 December 2000 and 31 March 2001.

\subsection{Data collection}

\subsubsection{Vaccination}

The status of vaccination and demographic variables were collected by public health nurses in each health center and was sent to Bureau of Health, Taipei county, for data coding and punching. Since some elderly people might have the uptake of vaccination outside the county, the status of vaccination was partially ascertained by linking target population with national health insurance data. Exposed to vaccination was defined as the uptake of vaccination between 1 October and 31 December 2000. The unexposed group was outside this period. Information collected included name, identification number, age, gender, residence, and date of vaccination.

\subsubsection{Risk group}

High-risk group was defined as those who were hospitalized or had outpatient visits between 1 October 1999 and 30 September 2000 attributed to the following causes: coronary heart disease (ICD-9-CM codes 393-398, 410-414, 425,428 , and 429), chronic lung disease (ICD-9-CM codes 491-496 and 500-518), diabetes mellitus (ICD-9-CM code 250), chronic renal disease (ICD-9-CM codes 581, 582, and 
585), vasculitis or rheumatologic disease (ICD-9-CM codes 446, 710, and 714), dementia or stroke (ICD-9-CM codes 290-294, 331, 340, 341, 348, and 438), non-hematologic cancer (ICD-9-CM codes 140-199), hematologic cancer (ICD9-CM codes 200-208), or the acquired immunodeficiency syndrome (ICD-9-CM codes 042-044) [12]. Other subjects without these diseases were defined as average-risk group.

\subsubsection{Medical utilization behavior}

Since the study was not a randomized trial, the comparison between the vaccinated group and the unvaccinated group with respect to death and hospitalization may be confounded by medical utilization behavior. For example, subjects who were admitted to hospital or to undergo health check-up may be more inclined to take vaccination than those who were not. Such a selection bias may affect the estimate of the benefit of vaccination. We therefore collected all-cause hospitalization data and history of health check-up before receiving vaccination a year ago. The "health check-up" was composed of a series of free health examination, including complete blood count, urinalysis, fasting plasma glucose, blood lipids, and provided by the health insurance for elderly people aged 65 years or older per year. The frequency of all-cause hospitalization was categorized into two groups, frequent $(\geq 2)$ and infrequent $(<2)$, according to the median value of frequency of hospitalization of all target subjects in the preceding year. The status of health check-up was coded as a binary variable (yes/no).

\subsection{Statistical analysis}

Multiple logistic regression models were used to calculate adjusted relative risk for complication and death with the adjustment for potential confounders, including age, gender, the frequency of outpatient department visit, the frequency of hospitalization, and the status of adult health check-up.

\subsection{Cost-effectiveness analysis}

Direct cost and indirect cost were collected in this study. Direct cost included vaccine cost, hospitalization cost due to complications, and cost caused by severe vaccine side-effect. Indirect cost consisted of traveling fee and production loss due to vaccination, severe vaccine side-effect, and hospitalization. Augmented costs involved in other hospitalizations not associated with influenza were also included. All the basecase parameters, assumptions, and detailed description used for the analysis are summarized in Table 4 and footnote of Table 5. Costs collected in this study were only limited to the study period between 1 December 2000 and 31 March 2001. To accommodate time preference, $5 \%$ discount rate was applied to calculate direct and indirect cost. The incremental cost-effectiveness ratio expressed by trading extra cost for per death averted or per life year gained for vaccination against non-vaccination was calculated [13].

\section{Results}

\subsection{Descriptive results}

Of 226,997 subjects aged 65 years or older, a total of 80,840 subjects had undergone influenza vaccination between 1 October and 31 December in 2000. The overall vaccination rate was $35.6 \%$.

Table 1 shows the comparison of baseline variables between the vaccinated group and the unvaccinated group. Both groups were dissimilar with respect to mean age $(P=0.007)$, gender $(P=0.001)$, high-risk group status $(P=0.001)$, the frequency of outpatient department visits $(P<0.001)$, the frequency of hospitalization $(P<0.001)$, and the uptake of adult health check-up of the preceding year $(P=0.001)$. The vaccinated groups were slightly younger, more likely to have concurrent major diseases at base line, and to exploit health care resources in the preceding year.

Similar findings were also noted whilst the comparison of baseline variables between the vaccinated group and the unvaccinated group was made by risk status. Except the frequency of hospitalization, both vaccinated and unvaccinated group were different with respect to other variables regardless of risk status.

Table 1

Characteristics by vaccination status

\begin{tabular}{|c|c|c|c|}
\hline Characteristics & Vaccinated & Unvaccinated & $P$ \\
\hline No. $(\%)$ & $80,840(35.6)$ & $146,157(64.4)$ & \\
\hline Age (mean, years-old) & 73.17 & 73.24 & 0.007 \\
\hline Age group (aged over $75, \%$ ) & 36.28 & 35.91 & 0.083 \\
\hline Gender (male, \%) & 56.87 & 52.85 & 0.001 \\
\hline High-risk status $(+, \%)$ & 52.58 & 32.89 & 0.001 \\
\hline No. of OPD ${ }^{\mathrm{a}}$ visit during the preceding year & 31.12 & 22.51 & $<0.001$ \\
\hline No. of hospitalization during the preceding year & 2.45 & 2.24 & $<0.001$ \\
\hline Proportion of highly-frequent OPD $(+, \%)^{\mathrm{b}}$ & 62.36 & 37.49 & 0.001 \\
\hline Proportion of highly-frequent hospitalization $(+, \%)^{\mathrm{c}}$ & 24.31 & 15.33 & 0.001 \\
\hline Participation rate of adult health check-up $(+, \%)^{\mathrm{d}}$ & 35.00 & 16.99 & 0.001 \\
\hline
\end{tabular}

\footnotetext{
a OPD: outpatient department.

b No. of OPD visiting greater than 20 times in the preceding year.

c No. of hospitalization greater than 2 in the preceding year.

${ }^{\mathrm{d}}$ Ever received the service of adult health check-up in the preceding 1 year.
} 
Table 2

Adjusted relative risk ${ }^{\mathrm{a}}$ of all-cause mortality and hospitalization for the vaccinated vs. the unvaccinated

\begin{tabular}{lll}
\hline & \multicolumn{2}{l}{ Adjusted RR $(95 \%$ CI $P$-value } \\
\cline { 2 - 3 } & All persons & High-risk group \\
\hline All-cause mortality & $0.71(0.65,0.77),<0.001$ & $0.67(0.61,0.74),<0.001$ \\
Hospitalization due to pneumonia and influenza & $0.85(0.76,0.96), 0.009$ & $0.89(0.77,1.03), 0.123$ \\
Hospitalization due to respiratory diseases & $1.01(0.94,1.09), 0.769$ & $0.64(0.54,0.75),<0.001$ \\
Hospitalization due to congestive heart failure & $0.98(0.87,1.09), 0.689$ & $0.72(0.57,0.89), 0.003$ \\
Hospitalization due to all diseases $^{b}$ & $0.98(0.92,1.05), 0.680$ & $0.89(0.79,1.01), 0.996$ \\
\end{tabular}

a Extraneous factors adjusted in the model include age, sex, the frequency of hospitalization, and the status of health check-up.

b All diseases include pneumonia, influenza, respiratory diseases, and congestive heart failure.

\subsection{The impact of vaccination on all-cause mortality and hospitalization by risk group}

Adjusted relative risks of all-cause mortality after adjusting for age group (age $\geq 75 /$ age $<75$ ), gender (male/female), the frequency of hospitalization $(\geq 2 /<2)$, and the status of health check-up (yes/no) of the preceding year are shown in Table 2 . The adjusted relative risk for the vaccinated versus the unvaccinated was 0.71 (95\% CI: $0.65-0.77)$, indicating a significant $29 \%$ (95\% CI: $23-35 \%, P<0.001$ ) reduction of all deaths for all subjects. The corresponding figures were 0.67 (95\% CI: 0.61-0.74) for the high-risk group and 0.64 (95\% CI: 0.54-0.75) for the average-risk group. This suggests 33\% (95\% CI: 26-39\%) and 36\% (95\% CI: $25-46 \%$ ) reductions of all deaths for high-risk group and average-risk group, respectively.

Table 2 also shows adjusted relative risks of hospitalization due to pneumonia and influenza, respiratory diseases, congestive heart failure and all diseases, respectively for the vaccinated versus the unvaccinated by risk group after controlling for age, sex, the frequency of hospitalization, and the status of health check-up. Vaccination against influenza in the reduction of hospitalization due to pneumonia and influenza, respiratory diseases and all diseases was only significant in average-risk group but not in high-risk group. A non-significant association was found for congestive heart failure both in average-risk and high-risk group.

\subsection{The impact of vaccination on all-cause mortality and hospitalization by baseline disease type}

Adjusted relative risks of all-cause mortality by baseline disease type, including pneumonia and influenza, type $2 \mathrm{di}-$ abetes mellitus, congestive heart failure, coronary heart disease, cerebrovascular disease, cancers, and chronic renal failure, are shown in Table 3. Influenza vaccination led to a significant reduction of all-cause mortality for all types of disease.

Adjusted relative risks of hospitalization due to all diseases by baseline disease type are also shown in Table 3 . The efficacy of vaccination in reducing hospitalization was small whereas vaccination significantly reduced hospitalization for persons free of any above-mentioned disease $(\mathrm{RR}=0.81,95 \% \mathrm{CI}: 0.66-0.99)$, which is very close to the estimate $(\mathrm{RR}=0.80,95 \% \mathrm{CI}: 0.70-0.91)$ for average-risk subject shown in Table 2.

\subsection{Cost-effectiveness analysis}

Table 4 shows the parameters and detailed calculation or description of direct cost and indirect cost involved in vaccination against influenza for the elderly persons.

Table 5 shows the results of the incremental costeffectiveness ratio for the comparison between the vaccinated group and the unvaccinated group for the elderly persons aged 65 years or older. By the application of $5 \%$ discount rate, the incremental cost-effectiveness ratio was US\$ 3899 (in 2001 US dollars) per death averted and US\$ 309 per life year gained. Taking augmented costs (costs for hospitalization due to other diseases) into account, the corresponding figures were US\$ 8749 , and 695 , respectively.

\section{Discussion}

A prospective study was designed to evaluate the efficacy and cost-effectiveness of a universal vaccination against in -

Table 3

Adjusted relative risk ${ }^{\mathrm{a}}$ of all-cause mortality and hospitalization due to all diseases for the vaccinated vs. the unvaccinated by baseline disease type

\begin{tabular}{lll}
\hline Baseline disease type & \multicolumn{2}{l}{ Adjusted RR $(95 \% \mathrm{CI}) P$-value } \\
\cline { 2 - 2 } & All-cause mortality \\
\hline Diabetes mellitus & $0.70(0.60,0.83),<0.001$ & $0.93(0.79,1.10), 0.383$ \\
Pneumonia and influenza & $0.64(0.54,0.77),<0.001$ & $0.94(0.83,1.06), 0.300$ \\
Congestive heart failure & $0.74(0.58,0.93), 0.011$ & $0.73(0.61,0.86),<0.001$ \\
Coronary heart disease & $0.79(0.68,0.92), 0.003$ & $0.94(0.84,1.04), 0.219$ \\
Cerebrovascular disease & $0.79(0.62,1.00), 0.051$ \\
Chronic renal failure & $0.54(0.44,0.66),<0.001$ & $0.90(0.80,1.02), 0.089$ \\
Cancers & & $0.86(0.71,1.04), 0.128$ \\
\end{tabular}

\footnotetext{
a Extraneous factors adjusted in the model include age, sex, frequency of hospitalization, and the status of health check-up.
} 
Table 4

Parameters for direct and indirect cost (in 2001 US dollars)

\begin{tabular}{|c|c|c|}
\hline Cost items & Base-case value & Detailed calculation or description \\
\hline \multicolumn{3}{|l|}{ Direct cost } \\
\hline Vaccination & US\$ 7.54 & Drug cost $($ US $\$ 4.32)+$ consultation cost $($ US\$ $\$ 2.88)+$ administration cost (US\$ 0.34$)$ \\
\hline Side-effect & US\$ 0.104 & $\begin{array}{l}\text { Payment for one outpatient treatment (US\$ } 11.53) \times \text { rate of side-effect due to vaccination }(1.8 \%) \\
{[25] \times \text { half of these need outpatient treatment }(0.5)}\end{array}$ \\
\hline Hospitalization & US\$ 3304.91 & Average cost of one admission \\
\hline \multicolumn{3}{|l|}{ Indirect cost } \\
\hline \multicolumn{3}{|l|}{ Production loss ${ }^{\mathrm{a}}$} \\
\hline Vaccination & US\$ 5.94 & $\begin{array}{l}\text { Half of person to accompany is required }(0.5) \times \text { time lost }(2 \mathrm{~h}) \times \text { production value per hour (US\$ } \\
5.94)^{\mathrm{b}}\end{array}$ \\
\hline Side-effect & US\$ 0.107 & $\begin{array}{l}\text { Rate of side effect due to vaccination }(1.8 \%) \times \text { half of persons to accompany is required }(0.5) \times \text { half } \\
\text { of outpatient visiting }(0.5) \times \text { time lost }(4 \mathrm{~h}) \times \text { production value per hour (US } \$ 5.94)\end{array}$ \\
\hline Hospitalization & US\$ 709.59 & $\begin{array}{l}\text { Average duration of admission }(19.91 \text { day }) \times \text { three-fourth of persons to accompany is required } \\
(0.75) \times \text { time lost }(8 \mathrm{~h} \text { per day }) \times \text { production value per hour }(\text { US } \$ 5.94)\end{array}$ \\
\hline \multicolumn{3}{|l|}{ Traveling fee } \\
\hline Vaccination & US\$ 1.5 & Patient with $1 / 2$ family accompanied ( 1.5 persons $) \times$ traveling cost (US\$ 1$)$ \\
\hline Side-effect & US\$ 0.0135 & $\begin{array}{l}\text { Side effect due to vaccination }(1.8 \%) \times \text { half of outpatient visiting }(0.5) \times \text { patient with } 1 / 2 \text { family } \\
\text { accompanied }(1.5 \text { persons }) \times \text { traveling cost }(\text { US } \$ 1)\end{array}$ \\
\hline Hositalization & US\$ 1.75 & Patient with $3 / 4$ family accompanied $(1.75$ persons $) \times$ traveling cost (US $\$ 1$ ) \\
\hline
\end{tabular}

${ }^{\text {a }}$ As we assume the productivity of a person aged over 65 years is null, production loss is attributed to care givers.

b Production value per hour $=$ average gross national product per person (US\$ 12,970)/[average monthly working hours for employer $(182 \mathrm{~h}) \times 12$ month] = US\$ 5.94.

fluenza for the elderly persons aged 65 years or older in one county of northern Taiwan in which approximately 230,000 elderly people dwell. In such a dense population, universal vaccination for the elderly people aged 65 years or older is of great service to stamp the outbreak of influenza, leading to influenza-like illness or death, because of herd immunity on the basis of evidence from several military trials conducted in the US since 1940 which showed the vaccination not only demonstrated 70-90\% efficacy in reducing influenza infection but also developed herd-immunity to prevent nonvaccinated population from infection with influenza during influenza epidemics $[14,15]$.

Our results on mortality reduction attributed to vaccination and cost-effectiveness analysis further uphold such universal vaccination against influenza for the elderly people in this area. Vaccination against influenza for the elderly persons can lead to a $29 \%$ (95\% CI: 23-35\%) significant reduction of all deaths. By risk group, it can lead to a 33\% (95\% CI: $26-39 \%$ ) and 36\% (95\% CI: 25-46\%) reduction of all deaths for the high-risk group and average-risk group, respectively. By disease type, vaccination can lead to $21-46 \%$ reduction of death $(P$-value $\leq 0.05)$ for subjects with diabetes mellitus, pneumonia, congestive heart failure, coronary heart disease, cerebrovascular disease, chronic renal failure or cancers, and $33 \%(P$-value $\leq 0.05)$ for others. Regarding economic evaluation, the extra cost incurred in vaccination was US\$ 3899 dollars to avert one death, and US\$ 309 to save an additional life year, respectively. Compared with other prevention program such as cancer screening with incremental cost-effectiveness ratio greater than US\$20,000, universal vaccination against influenza for the elderly people seems very cost-effective.

It is difficult to make the direct comparison of our all-cause mortality result with those from previous studies because the efficacy or effectiveness of influenza vaccines varies across studies as stated in a meta-analysis study by pooling a series of observational studies, giving the range of efficacy from 56 to $76 \%$ [1].

The heterogeneity of efficacy or effectiveness may be explained by viral strain, the period of study, and study design of evaluation [16]. For instance, the effectiveness of influenza vaccination in preventing pneumonia and influenza hospitalizations in Foster et al. [17] study was estimated as $45 \%$ during the peak of influenza activity but merely $21 \%$ during the period of dwindling influenza activity.

We find that vaccination was more efficacious for averagerisk group than high-risk group whatever it was aimed at preventing mortality or reducing hospitalization due to influenza and its complications. The finding that the influenza vaccination is more efficacious in average-risk subject was by no means innovative and was consistent with the previous results, reported by Barker and Mallooly [18], of an efficacy equal to $100 \%$ for the vaccinees without risk and negative for high-risk subjects during 1968-1969 season. The findings of the efficacy up to $100 \%$ both for subjects without risk and for high-risk persons were reported in the winter periods of 1972-1973. However, the interpretation of results should be taken with great caution because of small sample size in this perspective studies. Based on a large-scale prospective study, our study confirmed the finding that the efficacy of influenza vaccination is more efficacious for average-risk subjects. Such a finding suggests that high-risk subject may have poorer response to vaccine than average-risk subject as found in previous studies $[19,20]$.

Our study findings also show that there is no evidence that influenza vaccination is efficacious for elderly persons in reducing hospitalization. This finding is hard to explain. There 
Table 5

Cost-effectiveness analysis between vaccination and non-vaccination (augmented cost included)

\begin{tabular}{|c|c|c|c|c|c|c|c|c|c|c|c|}
\hline \multirow[t]{2}{*}{ Program } & \multicolumn{3}{|l|}{ Direct cost } & \multicolumn{3}{|l|}{ Indirect cost } & \multirow[t]{2}{*}{ Total } & \multicolumn{2}{|c|}{ Effectiveness } & \multicolumn{2}{|l|}{$\begin{array}{l}\text { Incremental } \\
\text { cost-effectiveness ratio }\end{array}$} \\
\hline & Vaccination & Hospitalization & Total & Vaccination & Hospitalization & Total & & Death & $\begin{array}{l}\text { Life-year } \\
\text { Gained }\end{array}$ & $\begin{array}{l}\text { Per death } \\
\text { averted } \\
\text { (discount) }\end{array}$ & $\begin{array}{l}\text { Per life-year } \\
\text { gained } \\
\text { (discount) }\end{array}$ \\
\hline $\begin{array}{l}\text { No } \\
\text { vaccination }^{\mathrm{a}}\end{array}$ & 0 & $15,004,093$ & $15,004,093$ & 0 & $3,229,440$ & $3,229,440$ & $18,233,534$ & 2610 & & & \\
\hline $\begin{array}{l}\text { No } \\
\text { vaccination } \\
\text { (augmented } \\
\text { cost included) }\end{array}$ & 0 & $42,251,916$ & $42,251,916$ & 0 & $9,871,475$ & $9,871,475$ & $52,123,391$ & & & & \\
\hline Vaccination $^{\mathrm{a}}$ & $1,735,165$ & $14,704,013$ & $16,439,178$ & $1,716,210$ & $3,164,850$ & $4,881,060$ & $21,320,239$ & 1856 & & & \\
\hline $\begin{array}{l}\text { Vaccination }^{\mathrm{b}} \\
\text { (augmented } \\
\text { cost included) }\end{array}$ & $1,735,165$ & $45,068,710$ & $46,803,875$ & $1,716,210$ & $10,529,573$ & $12,245,783$ & $59,049,658$ & & & & \\
\hline Vaccination-No & $1,735,165$ & $-300,080$ & $1,435,085$ & $1,716,210$ & $-64,590$ & $1,651,620$ & $3,086,705$ & 754 & 9,500 & U.S. 4,094 (U.S. 3,899) & U.S. 325 (U.S. 309) \\
\hline $\begin{array}{l}\text { vaccination } \\
\text { (augmented }\end{array}$ & $1,735,165$ & $2,816,794$ & $4,551,959$ & $1,716,210$ & 658,098 & $2,374,308$ & $6,926,267$ & 754 & 9,500 & U.S. 9,186 (U.S. 8,749) & U.S. 729 (U.S. 695) \\
\hline
\end{tabular}

a Parameters without taking augmented cost into account: admission rate for pneumonia and influenza, all respiratory diseases and congestive heart failure for non-vaccinated group $=0.02 ;$ mortality rate for all causes for non-vaccinated group $=0.0115$; admission rate for vaccinated group $=0.0196$; mortality rate for vaccinated group $=0.008177$; average cost of one admission due to pneumonia and influenza, all respiratory diseases, and congestive heart failure =US $\$ 3304.91$; average duration of one admission due to pneumonia and influenza, all respiratory diseases, and congestive heart failure = 19.91 days; average loss of productivity and traveling cost for one admission = US $\$ 711.34$; average life expectancy of 70-74 year-old people in Taiwan according to statistic data in $2000=12.6$ years.

b Parameters with taking augmented cost into account: all-cause admission rate for non-vaccinated group $=0.075$; all-cause mortality rate for non-vaccinated group $=0.0115$; all-cause admission rate for vaccinated group $=0.800$; all-cause mortality rate for vaccinated group $=0.008177$; average cost of one admission due to all causes $=$ US $\$ 2481.79$; average duration of one admission due to all causes $=16.22$ days; average loss of productivity and traveling cost for one admission due to all causes $=$ US $\$ 579.83$. 
is one possibility accounting for this finding. It is well known that the elderly people may be infected with influenza by contacting with children. It is probable that the most benefit of influenza vaccination for average-risk subject aged 65 years or older may protect the elderly people from being infected with influenza from children. On the contrary, it is customary for Chinese people to separate children from the elderly people with illness, particularly pneumonia. Hence, the efficacy of influenza vaccination in reducing hospitalization may be discounted.

Our results of cost-effectiveness analysis were also more conservative than those in the previous studies [21-23]. The net cost per life-year gained in Riddiough et al. study was US\$ 13 for medical cost associated with influenza as compared with the corresponding figures US $\$ 309$ in our study (Table 5) [22]. The explanation for the disparity was partially due to the inclusion of average-risk group in our study and partially due to the exclusion of indirect cost in Riddiough et al. study.

Since our study design was not based on a randomized trial, this may introduce selection bias while the mortality and hospitalization between the vaccinated group and the unvaccinated group was compared. To tackle this bias, several factors were taken into account, including age, sex, the frequency of hospitalization, the frequency of outpatient visit, and the propensity for the uptake of health check-up. It can be seen that the crude efficacy for preventing all-cause deaths was only $8 \%$ but the adjusted efficacy was raised to $29 \%$ after controlling these factors. This underscored the importance of the adjustment for factors leading to selection bias.

In contrast to other previous studies, our study also took account of baseline disease type while the efficacy of vaccination was evaluated. Our study found subjects free of serious diseases gained the most benefit from vaccination. This finding can also uphold the better efficacy found in average-risk group.

There were two merits of cost-effectiveness analysis in our study. The indirect cost due to the accompanied for patients with complication was taken into account in our study. This has been rarely addressed in the previous study. In addition, augment cost, i.e. cost for treating other disease was also considered in this study. The aspect of augment cost was particularly important for vaccination in the elderly people because these subjects may be more likely to be susceptible to other diseases even if complications caused by influenza can be prevented. Hence, it is improbable that a policy of expanding vaccination to cover all elderly could represent a cost-saving intervention. Such an interpretation should be taken with great caution because the meaning of augment costs may have different significance in different countries. For example, augment cost may have more significant implication in the US than UK as discussed in Nicholson et al. [6].

There were two limitations in our study. First, we did not consider the outcome with respect to outpatient visits because we were not sure how accurate this information could be representative of the cause of visits associated with influenza.
Second, the calculation of indirect cost was based on capital approach. This may raise a contingent value of life. To tackle this philosophical debate, the willingness to pay approach may be undertaken in the future study [24].

In conclusion, immunization against influenza for the elderly people aged 65 years or older may be not only important to high-risk group but also effective in average-risk group in terms of mortality reduction. Universal vaccination against influenza for the elderly people seems very cost-effective. However, a significant benefit of reducing hospitalization can be only seen in average-risk subjects but not in high-risk group subjects.

\section{References}

[1] Gross PA, Hermogenes AW, Sacks HS, Lau J, Levandowski RA. The efficacy of influenza vaccine in elderly persons. A meta-analysis and review of the literature. Ann Intern Med 1995;123:518-27.

[2] She SM, Ho BS, She YC, et al. The efficacy of influenza vaccine in elderly persons in Taiwan. Taiwan, ROC: Center for Disease Control; 1999.

[3] Hak E, Nordin J, Wei F. Influence of high-risk medical conditions on the effectiveness of influenza vaccination among elderly members of 3 large managed-care organizations. Clin Infect Dis 2002;35:370-7.

[4] Nichol KL, Nordin J, Mullooly J, Lask R, Fillbrandt K, Iwane M. Influenza vaccination and reduction in hospitalizations for cardiac disease and stroke among the elderly. N Engl J Med 2003;348:132232.

[5] Govaert ME, Thijs CTMCN, Masurel N, Sprenger MJW, Dinant GJ, Knottnerus JA. The efficacy of influenza vaccination in elderly individuals. JAMA 1994;272:1661-5.

[6] Nicholson KG. Socioeconomics of influenza and influenza vaccination in Europe. Pharmacoeconmics 1996;9(Suppl 3):75-8.

[7] Wang CS, Wang ST, Chou PS. Efficacy and cost-effectiveness of influenza vaccination of the elderly in a densely populated and unvaccinated community. Vaccine 2002;20:2494-9.

[8] McBean AM, Babish JD, Warren JL. The impact and cost of influenza in the elderly. Arch Intern Med 1993;153:2105-11.

[9] Statistics of Communicable diseases and surveillance report in Taiwan area, 2000. Taiwan: Center for Disease Control, ROC; 2002. p. 52-7.

[10] Influenza: United States, 1989-90 and 1990-91 seasons. MMWR Morb Mortal Wkly Rep 1992;41:35-46.

[11] Statistics of communicable diseases and surveillance report in Taiwan area, 2000. Taiwan: Center for Disease Control, ROC; 2002. p. 44-6.

[12] Prevention and control of influenza: part I, vaccines: recommendations of the Advisory Committee on Immunization Practices (ACIP). MMWR Morb Mortal Wkly Rep 1994;43:1-13.

[13] Fedson DS. Evaluating the impact of influenza vaccination. Pharmaco-Economics 1996;9(Suppl 3):54-61.

[14] Davenport FM. Control of influenza, symposium on influenza. Med J Aust Spec 1973;(Suppl 1):33-8.

[15] Monto AS, Olazabel Jr F. Asian influenza in the Panama Canal Zone: isolation of a virus variant and protective effect of a vaccine containing A2/Japan/305/57. Am J Epidemiol 1966;83:101-12.

[16] Monto AS. The clinical efficacy of influenza vaccination. PharmacoEconomics 1996;9(Suppl 3):16-22.

[17] Foster DA, Talsma A, Furumoto-Dawson A, et al. Influenza vaccine effectiveness in preventing hospitalization for pneumonia in the elderly. Am J Epidemiol 1992;136:296-307.

[18] Barker WH, Mullooly JP. Impact of epidemic type A influenza in a defined adult population. JAMA 1980;244:2547-9. 
[19] Gross PA, Quinnan Jr GV, Weksler ME. Relation of chronic disease and immune response to influenza vaccine in the elderly. Vaccine 1989;7:303-8.

[20] Phair J, Kauffman CA, Bjornson A. Failure to respond to influenza vaccine in the aged: correlation with B-cell number and function. $\mathrm{J}$ Lab Clin Med 1978;92:822-8.

[21] Nichol KL, Margolis KL, Wuorenma J, Sternberg TV. The efficacy and cost effectiveness of vaccination against influenza among elderly persons living in the community. N Engl J Med 1994;331:778-84.

[22] Riddiough MA, Sisk JE, Bell JC. Influenza vaccination: costeffectiveness and public policy. JAMA 1983;249:3189-95.
[23] Mullooly JP, Bennett MD, Hornbrook MC, Barker WH, Williams WW, Patriarca PA, et al. Influenza vaccination programs for elderly persons: cost-effectiveness in a health maintenance organization. Ann Intern Med 1994;121:47-952.

[24] Birch S, Gafni A, O'Brien B. Willingness to pay and the valuation of programmes for the prevention and control of influenza. Pharmacoeconomics 1999;16(Suppl 1):55-61.

[25] Huang KC, Jan CF, Kao CL, Liu YL, Chen CY, Lin RS, et al. A pilot study of immune response after influenza vaccination. Formosan $\mathrm{J}$ Med 1999;3:661-6. 